\title{
Effectiveness of a Commercially Available Orthotic Insert
}

Sonia N Young ${ }^{*}$, Harvey W Wallmann, Allison Baston, Wes Smith and Kirsti Vittitow

College of Health and Human Services, Western Kentucky University, Bowling Green, Kentucky, USA

*Corresponding author: Sonia N. Young, Doctor of Physical Therapy Program, College of Health and Human Services, Western Kentucky University, Bowling Green, Kentucky, USA, Tel: (270) 745-3233; Fax: (270) 745-3497; E-mail: sonia.young@wku.edu

Rec date: Jan 31, 2016; Acc date: Mar 03, 2016; Pub date: Mar 10, 2016

Copyright: (C) 2016 Young SN, et al. This is an open-access article distributed under the terms of the Creative Commons Attribution License, which permits unrestricted use, distribution, and reproduction in any medium, provided the original author and source are credited.

\begin{abstract}
Background: Foot pain is a non-specific complaint among individuals who stand for extended periods of time. Prefabricated inserts have been effective for increasing balance and managing pain. One manufacturer has developed a line of general inserts for that purpose. The purpose of this study was to examine the effectiveness of a commercially available orthotic insert on balance, pain, and plantar foot pressures as well as to determine if enhancing balance may decrease pain.
\end{abstract}

Methods: Twenty-three subjects with at least 6 months non-specific foot pain participated and were issued a commercially available orthotic insert after screening. The Sensory Organization Test composite score was used to assess balance on the NeuroCom® Balance Manager SMART EquiTest. Foot Function Index (FFI) and Numeric Pain Rating Scale (NPRS) were used to assess pain. GAITRite was utilized to assess plantar foot pressures.

Results: Foot pain was decreased after one week with use of the commercially available orthotic insert. Increased balance scores were noted between day 1 without inserts and after day 7 of insert wear and also between day 1 with inserts and day 7 . Decreases in pain were noted between pre balance testing without inserts (day 1) and after day 7 as well as immediately after balance testing with inserts (day 1 ) and day 7 . Among the females, right lateromedial foot pressures increased from day 1 without inserts and immediately after the addition of inserts. An inverse relationship was observed between the NPRS and the composite balance scores after 1 week of insert wear.

Conclusions: Results indicate that a commercially available orthotic insert may be effective in managing foot pain and increasing balance after one week. Additionally, inserts appear to shift the right foot pressures from the predominately medial aspect of the foot to the lateral aspect of the foot for females but not males.

Keywords: Balance; Foot pain; Orthotics; Foot pressures; Foot function index (FFI); Numeric pain rating scale (NPRS)

\section{Introduction}

Non-specific foot pain is a common complaint among individuals who stand for extended periods of time [1]. Inserts are often prescribed to reduce non-specific foot pain in daily activities [2]. The use of well-fitting orthotic inserts can help redistribute pressure on the foot and improve balance, and therefore may decrease pain [3]. However, there is no clear information to help determine the effectiveness of an insert. One manufacturer has developed a line of general inserts designed to properly fit each individual based on arch height and body mass index (BMI). There are two types of orthotic inserts. In this study, prefabricated inserts refer to those that can be purchased over-the-counter at a local retail store. Custom-made orthotic inserts are defined as those that have been molded to an individual's foot by a certified clinician. Research has found no difference in plantar pressures when comparing contoured prefabricated and custom-made inserts [4]. Stolwijk et al. [3] concluded that although custom made insoles were effective in reducing peak pressure and average pressure, there was not a greater difference in plantar pressure redistribution between insole designs. As such, they advised having a general insole design that can adapt according to foot complaints and arch height [3].
Arch height can also be an important factor in distribution of plantar pressures. Higher arch height is associated with greater lateral forefoot pressure when walking [5] and standing [6]. Lower arch individuals may have increased pressure under the midfoot [6] or hallux and medial mid-foot [7]. Hegedus et al. [8] found that longitudinal arch height does not affect pain. However, high BMI has also been found to influence height of arches [6]. Ankle instability can increase the risk of falls leading to sprains, fractures, and chronic problems. Properly fitted orthotics can increase balance as can the addition of simple exercises [9-11]. Research has reported advantages of inserts for increasing balance, but few studies have compared the effectiveness of these different inserts [12]. Non-specific foot pain negatively affects a person's ability to perform activities of daily living $[1,2,13]$. A study suggests that custom-made foot orthoses are effective for pain reduction in the pes cavus foot [14]. A study has found inserts useful in reducing pain on walking and improving activity of daily living performance [15]. Conversely, other studies have not shown significant difference in the use of custom-made orthotics to decrease pain $[1,16]$. While research supports the use of inserts for pathologies causing foot pain such as rheumatoid arthritis [13] and plantar fasciitis $[15,16]$, there is limited support for the effectiveness of inserts for nonspecific foot pain [3]. The purpose of this study was to determine the effectiveness of a commercially available orthotic insert on plantar foot pressure, balance, and pain. The authors hypothesized that pain would be decreased, plantar pressures would be distributed more efficiently, 
and balance would be increased. It was additionally hypothesized that enhancing balance may decrease pain.

\section{Methods}

\section{Design}

A single group, quasi-experimental, within-subjects design using one way ANOVA repeated measures was used to determine the effectiveness of Dr. Scholl's Custom Fit orthotic inserts on plantar foot pressures, balance, and pain on walking and balance. Paired samples ttest was performed for Functional Foot Index (FFI) day 1 versus day 7. Pain and balance were assessed pre- and post-inserts and 1 week after insert wear utilizing the Numeric Pain Rating Scale (NPRS) and the Sensory Organization Test (SOT), respectively. A one-way repeated measures ANOVA was used to analyze balance and pain differences with the NPRS, and differences between right and left lateromedial $(\mathrm{L} / \mathrm{M})$ foot pressures for males and females. Spearman's rho $(\rho)$ was used to assess the relationship between the NPRS and the balance scores after 1 week of insert wear.

\section{Subjects}

Twenty-three subjects (10 males and 13 females) at least 18 years of age, mean age 34.2 years $(+12.4 \mathrm{SD})$, with at least 6 months foot pain participated. Subjects were volunteers from a sample of convenience from the local Medical Center and the general population in Bowling Green, Kentucky. A screening questionnaire was given to determine each individual's health status and ability to participate based on the inclusion and exclusion criteria. Inclusion criteria for subjects consisted of adults with the following: non-specific foot pain for at least 6 months, ability to ambulate without an assistive device, and English speaking. Subjects were excluded from the study if they were currently wearing Dr. Scholl's ${ }^{\oplus}$ Custom Fit ${ }^{\circledR}$ orthotic inserts, had acute pain or trauma within the last 6 months, had uncontrolled hypertension, had recently experienced vertigo, were currently taking medication that could affect balance, or had been diagnosed with any vestibular pathology.

Each subject was assured that all personal information and collected data would remain confidential by the use of a number code versus using the subject's names. The study was approved by the Institutional Review Board at Western Kentucky University prior to written informed consent and testing for each subject.

\section{Instrumentation}

GAITRite- CIR Systems, Inc. ${ }^{\circledR}$ GAITRite System measures temporal (timing) and spatial (distance) parameters via an electronic walkway connected to the USB port of a Windows ${ }^{\oplus} \mathrm{XP} / \mathrm{Vista} / 7$ personal computer and has been reported to be valid and reliable with good test- retest reliability $[17,18]$. The walkway is 16 feet long, 2 feet wide, and contains 18,432 sensors. With ambulation on the walkway, the system captures the geometry and the relative arrangement of each footfall as a function of time. The application software controls the functionality of the walkway and processes the raw data into footfall patterns. Plantar pressure measurements evaluated were integrated pressure over time, peak time, active area, and peak pressure.

\section{SMART balance manager}

The NeuroCom ${ }^{\circledR}$ Balance Manager-SMART EquiTest SOT was used to measure the subject's ability to control center of gravity in a variety of conditions. This machine is a three-sided booth with a moveable force plate, monitor, and safety harness for the subject. Both the booth and the force plate are sway-referenced, meaning they are sensitive to the person's sway and will move according to how much sway the person undergoes during different conditions of the SOT [19].

\section{Foot function index (FFI)}

The FFI is a 20 item questionnaire assessing 3 subscales: pain (6 questions), disability ( 9 questions), and activity limitation (5 questions) [20]. The FFI was used to assess foot pain for each subject over the course of one week rather than a specific moment in time. In this study, only the pain subsection was utilized. It was scored on a 10 centimeter visual analog scale with verbal anchors of 0 'no pain' and 10 'worst pain imaginable.' A total of 60 represents the worst pain possible on this subscale. The scores for the 6 pain items were measured with a tape measure, added up, and divided by 60 to give the individual's cumulative pain score. For this index, a higher score indicates worsening foot health on a $0-100 \%$ range. The FFI is a self-report outcome measure which took the subjects approximately 5 minutes to complete; the FFI has been shown to have a high test-retest reliability (ICC $=0.87$ ) and high internal reliability (Cronbach's alpha=0.96) [20].

\section{Numeric pain rating scale (NPRS)}

The NPRS is a frequently utilized measurement of pain. This tool consists of a verbal pain rating on a $0-10$ scale. The 0 rating was described as having "no pain at all." The 10 rating was described as having "worst imaginable pain." The minimally clinical important difference (MCID) for this tool was shown to be 2 points on average with a raw reduction of $30 \%$ for chronic pain [21]. A 1 point reduction of pain has shown to be consistent with a $15 \%$ reduction in chronic pain [22]. In that same study, a 2 point reduction correlated with a $33 \%$ change. This was associated with the concept of "much better" improvement in chronic pain.

\section{Procedures}

After initial screening and completion of informed consent, each subject who met the inclusion criteria was instructed to go to a local retail store to utilize the Dr. Scholl's ${ }^{\circledR}$ FootMapping ${ }^{\circledR}$ Kiosk. The Kiosk provided each subject a Custom Fit $^{\oplus}$ orthotic insert number based on arch height, body type, and shoe size [25]. An orthotic insert was then issued to each subject by the researchers based on this number on day 1 of testing.

On day 1 of testing, each subject was given a questionnaire to determine the effects of foot pain on daily activities and then instructed to rate current pain using the NPRS. Using a counterbalanced order, balance and gait assessments were conducted using the Balance Manager and GAITRite scale, respectively.

Footfall pressures while walking on the GAITRite were used to measure plantar foot pressures (Figure 1). 
Page 3 of 5

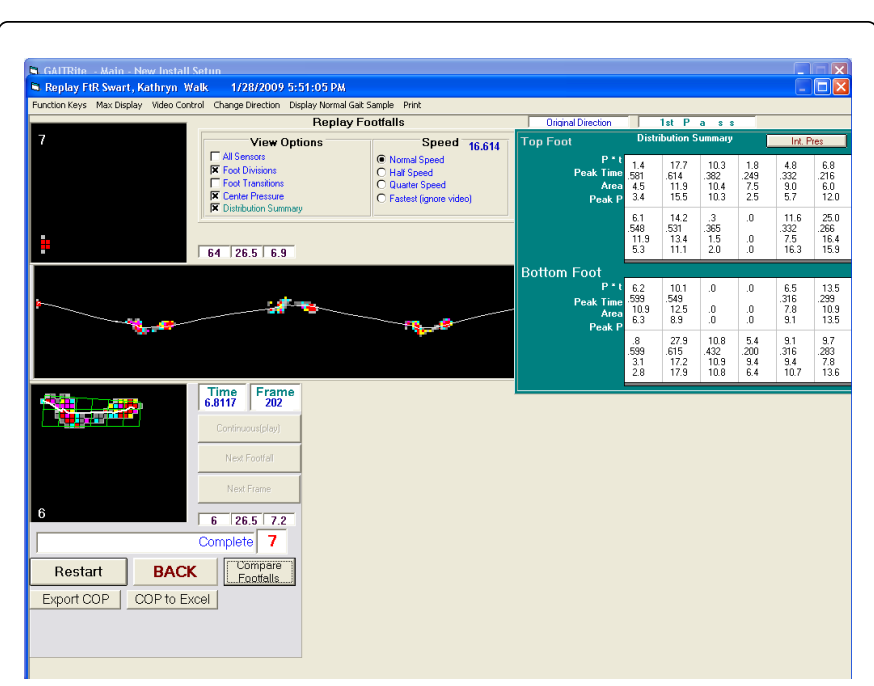

Figure 1: GAITRite software displaying foot pressure data. Average lateromedial peak pressure ratios were calculated from the data within the 2 sets of grids on the right.

Each subject was instructed to stand behind the GAITRite approximately 2 meters then ambulate across the walkway at a comfortable speed to a set stopping point placed 1 meter beyond the mat. Each was given two trials, the first one being considered as practice.

Balance was assessed using the SOT, which utilizes a computerized system to evaluate sensory interactions in standing balance [23]. The SOT, which has been determined to have good test-retest reliability [24], provides valuable information about whether an individual can use inputs from the visual, proprioceptive, and vestibular systems to maintain balance as well as suppress inaccurate sensory information. To perform this test, subjects were asked to step onto the platform with their shoes on and progress through the 6 conditions (Figure 2).

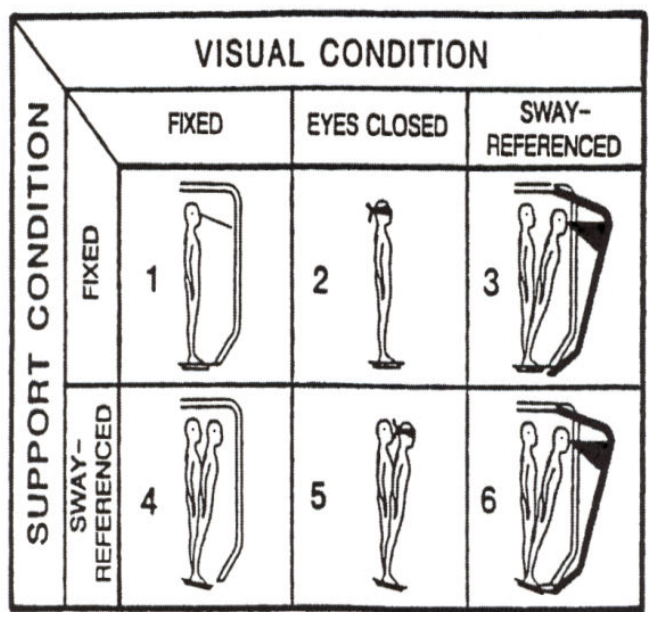

Figure 2: The 6 Sensory Organization Test (SOT) testing conditions used with the NeuroCom Smart Balance Master System. Image from NeuroCom International.
They were instructed to maintain an upright posture as much as possible. Three trials of each condition were performed lasting 20 seconds per trial. Subjects were given 30 second rest period between conditions. Subjects and testers were blinded to the scores until data analysis was completed.

The FFI was administered pre inserts before testing and then again post inserts on day 7 before testing. Before and after each balance test, pain was assessed using the NPRS. After obtaining the recommended prefabricated insert from a local Dr. Scholl's ${ }^{\circledR}$ Custom Fit ${ }^{\circledR}$ kiosk (Figure 3 ), the second tests were performed as described for baseline testing except that subjects were wearing the inserts to determine the immediate effects of the inserts.

Subjects were instructed to wear the recommended inserts as much as possible for 7 days following initial testing, keeping a subjective log of hours and comments. On day 7, the subject returned for the third session and completed the FFI; the subject then began with the first test, pain was reassessed, the next test was completed, and the final pain was assessed. Table 1 describes the testing process for all test sessions.

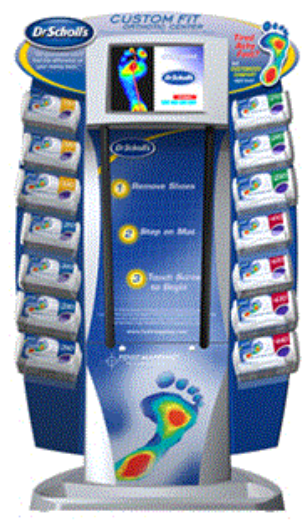

Figure 3: Prefabricated insert from a local Dr. Scholl's ${ }^{\star}$ Custom Fit $^{\circledR}$ kiosk

\begin{tabular}{|l|l|l|}
\hline Day 1/ pre-inserts & Day 1/ post-inserts & $\begin{array}{l}\text { Day 7/post-inserts 1 } \\
\text { week }\end{array}$ \\
\hline Foot Function Index & & Foot Function Index \\
\hline Pain & & Pain \\
\hline Balance Master & Balance Master & Balance Master \\
\hline Pain & Pain & Pain \\
\hline GAITRite & GAITRite & GAITRite \\
\hline Kiosk & & \\
\hline
\end{tabular}

Table 1: Example layout of testing scheme.

\section{Data Analysis}

This study utilized a quasi-experimental design to determine effectiveness of commercially available inserts on pain, plantar foot pressures, and balance. Since the study was measuring within-subjects for 3 different dependent variables, 3 different one way repeated 
measure ANOVAs were performed to determine statistical differences between groups. The sample size was determined utilizing an effect size of 0.40 , a power of 0.80 and 2 degrees of freedom (since there were 2 independent variables and time had 2 levels). It was concluded that 21 subjects were needed plus an additional 4 to account for a $20 \%$ attrition rate. All data were analyzed using SPSS 21.0 [26]. Foot

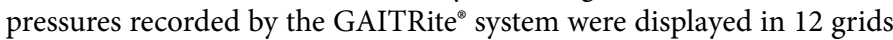
representing different areas of the foot (Figure 1). Medial and lateral peak pressure data were analyzed from the 6 grids representing the medial and lateral sides of each footprint to represent the amount of pressure displayed during pronation and supination, respectively. From the multiple footprints, averages were calculated for lateromedial peak pressure ratios.

\section{Results}

FFI pain differences were observed initially $(45.47, \mathrm{SD}=17.13)$ and after 1 week of insert wear $(28.46, \mathrm{SD}=20.99), \mathrm{t} 22=3.66, \mathrm{P}=0.001$. Balance scores differed, F2,44=16.071, $\mathrm{P}<0.0005$ with increased scores noted between day 1 without inserts (73.26, SD 6.59) and after day 7 of insert wear $(77.61, \mathrm{SD} 6.97, \mathrm{P}<0.000)$ and also between day 1 with inserts (72.87, SD 9.12) and day $7(\mathrm{P}<0.000)$. A one-way repeated measures ANOVA indicated decreases in NPRS values (F2,44=8.745, $\mathrm{P}=0.001)$ between pre balance testing without inserts (day 1) $(2.65, \mathrm{SD}$ 1.97) and pain after day 7 balance testing with inserts (1.35, SD 1.43 , $\mathrm{P}=0.002$ ) as well as between pain after balance testing with inserts (day 1) $(2.22, \mathrm{SD} 1.83)$ and day $7(\mathrm{P}=0.011)$. Right $\mathrm{L} / \mathrm{M}$ foot pressures increased for females $(\mathrm{F} 2,24=5.10, \mathrm{P}=0.014)$ from day 1 without inserts (1.03, SD 0.16) and with addition of inserts (day 1) (1.20, SD .20, $\mathrm{P}=0.04)$. An inverse relationship using Spearman's rho $(\rho(21)=-.426$, $\mathrm{P}=0.043$ ) was observed between the NPRS and the SOT composite balance scores after 1 week of insert wear.

\section{Discussion}

The purpose of this study was to determine the effectiveness of a commercially available orthotic insert on plantar foot pressure, balance, and pain. Results indicate that the Dr. Scholl's ${ }^{\otimes}$ Custom Fit ${ }^{\circ}$ orthotic inserts may be effective in managing foot pain and increasing balance after one week as balance improved and pain decreased as well as improving right lateromedial plantar pressures in females, suggesting a decrease in foot pronation.

Previous literature has examined the effectiveness of custom-made orthotic use on pain. Foot pain has been shown to decrease with the prescription of custom-made foot orthoses [14].

The current study supported this finding, with a significant reduction in pain on the NPRS at a single point in time and on the FFI looking at a week-long assessment. Amer et al. [2], determined that insole use is an effective treatment for women to reduce pain severity and improve daily activities. However, in that study, men showed no statistical significance in pain reduction. This is refuted in the present study as both men and women had a significant decline in pain and improved function with the addition of inserts. In contrast to a study performed by Zhang [1] in which subjects did not report significant pain relief within 6 weeks wearing custom-made orthotics, subjects wearing Dr. Scholl's ${ }^{\otimes}$ Custom Fit $^{\bullet}$ orthotic inserts reported improved pain levels within 1 week. However, pain in the study by Zhang was minimal at the beginning of the study allowing for no significant improvement with addition of inserts.
In a study performed by Gross et al. [27], the authors concluded that custom-made semi- rigid foot orthoses improved static and dynamic standing balance in older individuals. However, as opposed to the current study, Gross et al. [27] found no significant improvement in follow-up balance scores after each subject had worn the orthoses for 2 weeks. Additionally, they found a significant improvement in balance scores with the immediate insertion of orthoses. Mattacola et al. [28] found that custom-made orthoses were beneficial in improving postural sway and bilateral stance equilibrium when using the NeuroCom Smart Balance Master@. Their research also revealed that custom-made orthoses improved balance in individuals with rearfoot malalignment over time. Although rearfoot alignment was not measured in the current study, balance scores improved in subjects after wearing Dr. Scholl's Custom Fit Orthotics for one week.

By increasing lateromedial pressure, the right foot revealed a decrease in medial plantar foot pressure, thereby potentially alleviating a possible over-pronated position. Farzadi et al. [29] found medial arch support from prefabricated orthosis decreased the peak pressure and maximal force under the hallux, first metatarsal, and metatarsals 3-5 in one month in females. In agreement to this current study, Farzadi et al. [29] further discussed the redistribution of medial plantar pressures preventing possible pronated pathologies including hallux valgus. Redmond et al. [4] also found a reduction of medial pressures specifically the medial forefoot by approximately $20 \%$ with usage of prefabricated as well as custom-made orthotics. However, unlike the present study, Redmond et al. [4] did not assess patient-related factors such as pain. In agreement with this study, Aminian et al. [30] found that plantar pressures can be redistributed efficiently with immediate medial longitudinal arch support in prefabricated insoles.

Findings of this study will improve the clinical knowledge of the effectiveness of a commercially available orthotic insert on increasing balance, decreasing pain, and redistributing plantar foot pressures from medial to lateral. Dr. Scholl's ${ }^{\circledR}$ created general insert categories with their Custom Fit $^{\ominus}$ orthotic inserts based on arch height and BMI similar to a previous research suggestion by Stolwijk et al. [3] to have a few general designs for non-specific foot pain complaints. This allows patients the opportunity to purchase a customizable insert at a prefabricated insert cost. Redmond et al. [4] also suggested that prefabricated insoles have the potential to be as effective as custommade orthotics with a lesser cost.

Limitations included potential for learning effects as well as carryover effects [31]. The subjects were provided a practice trial with the different instrumentations to reduce potential of learning effects. Additionally, results did not indicate a significant difference in balance trials occurring in the same day. An attempt was made to decrease the impact of the carryover effect by allowing one week between day 1 and day 7 , however, more time could have been allowed between day 1 without inserts and day 1 with inserts.

Future research needs to be continued to improve the validity of this study. A larger sample size should be gathered to determine if a difference in the effectiveness of these inserts on pressure can be reproduced for the male population. BMI should also be assessed to recognize any significance on pain reduction, pressure distribution, and improved balance with a large or small body type and the addition of a commercially available orthotic insert. Arch height may have a hand in predicting the success of these inserts for people with a high arch versus a low arch, which should also be examined with future studies. Future research should include pre and post resting foot alignment measurement to confirm if the subjects were predominately 
pronators. Additional research is necessary to determine the long-term effectiveness of a commercially available insert.

\section{Conclusion}

Results indicate that the Dr. Scholl's ${ }^{\oplus}$ Custom Fit ${ }^{\circledR}$ orthotic inserts may be effective in managing foot pain and increasing balance after one week as balance improved and pain decreased. Additionally, right foot pressures appeared to change from that of medially dominated pressure to lateral after addition of inserts for females. This research suggests that individuals who have been experiencing foot pain may have a decrease in pain and more efficient balance when utilizing a commercially available orthotic insert.

\section{Acknowledgement}

A student research scholarship in the amount of $\$ 500$ was awarded from the Western Kentucky University College of Health and Human Services to help defray the cost of orthotic inserts.

\section{References}

1. Zhang J (2005) Chiropractic adjustments and orthotics reduced symptoms for standing workers. J Chiropr Med 4: 177-181.

2. Amer AO, Jarl GM, Hermansson LN (2014) The effect of insoles on foot pain and daily activities. Prosthet Orthot Int 38: 474-480.

3. Stolwijk NM, Louwerens JW, Nienhuis B, Duysens J, Keijsers NL (2011) Plantar pressure with and without custom insoles in patients with common foot complaints. Foot Ankle Int 32: 57-65.

4. Redmond A, Landorf KB, Keenan A (2009) Contoured, prefabricated foot orthoses demonstrate comparable mechanical properties to contoured, customized foot orthoses: A plantar pressure study. J Foot Ankle Res 2: 20.

5. Teyhen DS, Stoltenberg BE, Collinsworth KM, Giesel CL, Williams DG, et al. (2009) Dynamic plantar pressure parameters associated with static arch height index during gait. Clin Biomech (Bristol, Avon) 24: 391-396.

6. Periyasamy R, Anand S (2013) The effect of foot arch on plantar pressure distribution during standing. J Med Eng Technol 37: 342-347.

7. Jonely H, Brismée JM, Sizer PS Jr, James CR (2011) Relationships between clinical measures of static foot posture and plantar pressure during static standing and walking. Clin Biomech (Bristol, Avon) 26: 873-879.

8. Hegedus EJ, Cook C, Fiander C, Wright A (2010) Measures of arch height and their relationship to pain and dysfunction in people with lower limb impairments. Physiother Res Int 15: 160-166.

9. Bateni H (2013) Changes of postural steadiness following use of prefabricated orthotic insoles. J Appl Biomech 29: 174-179.

10. Hamlyn C, Docherty CL, Klossner J (2012) Orthotic intervention and postural stability in participants with functional ankle instability after an accommodation period. J Athl Train 47: 130-135.

11. Landry S, Nigg B, Tecante K (2010) Standing in an unstable shoe increases postural sway and muscle activity of selected smaller extrinsic foot muscles. Gait Posture 32: 215-219.

12. Christovão TC, Neto HP, Grecco LA, Ferreira LA, de Moura RCF, et al (2013) Effect of different insoles on postural balance: a systematic review. J Phys Ther Sci 25: 1353-1356.

13. Conrad KJ, Budiman-Mak E, Roach KE, Hedeker D, Caraballada R, et al (1996) Impacts of foot orthoses on pain and disability in rheumatoid arthritics. J Clin Epidemiol 49: 1-7.
14. Burns J, Crosbie J, Ouvrier R, Hunt A (2006) Effective orthotic therapy for the painful cavus foot: a randomized controlled trial. J Am Podiatr Med Assoc 96: 205-211.

15. Gross MT, Byers JM, Krafft JL, Lackey EJ, Melton KM (2002) The impact of custom semirigid foot orthotics on pain and disability for individuals with plantar fasciitis. J Orthop Sports Phys Ther 32: 149-157.

16. Landorf KB, Keenan AM, Herbert RD (2006) Effectiveness of foot orthoses to treat plantar fasciitis: a randomized trial. Arch Intern Med 166: 1305-1310.

17. McDonough AL, Batavia M, Chen FC, Kwon S, Ziai J (2001) The validity and reliability of the GAITRite system's measurements: A preliminary evaluation. Arch Phys Med Rehabil 82: 419-425.

18. Kuys SS, Brauer SG, Ada L (2011) Test-retest reliability of the GAITRite system in people with stroke undergoing rehabilitation. Disabil Rehabil 33: $1848-1853$

19. Wallmann HW, Gillis CB, Alpert PT, Miller SK (2009) The effect of a senior jazz dance class on static balance in healthy women over 50 years of age: a pilot study. Biol Res Nurs 10: 257-266.

20. Riskowski JL, Hagedorn TJ, Hannan MT (2011) Measures of foot function, foot health, and foot pain: American Academy of Orthopedic Surgeons Lower Limb Outcomes Assessment: Foot and Ankle Module (AAOS-FAM), Bristol Foot Score (BFS), Revised Foot Function Index (FFI-R), Foot Health Status Questionnaire (FHSQ), Manchester Foot Pain and Disability Index (MFPDI), Podiatric Health Questionnaire (PHQ), and Rowan Foot Pain Assessment (ROFPAQ). Arthritis Care Res (Hoboken) 63: S229-239.

21. Farrar J, Young JP, LaMoreaux L, Werth JL, Poole MR (2001) Clinical importance of changes in chronic pain intensity measured on an 11-point numerical pain rating scale. Pain 94: 149-158

22. Salaffi F, Stancati A, Silvestri CA, Ciapetti A, Grassi W (2004) Minimal clinically important changes in chronic musculoskeletal pain intensity measured on a numerical rating scale. Eur J Pain 8: 283-291.

23. Pang MY, Lam FM, Wong GH, Au IH, Chow DL (2011) Balance performance in head-shake computerized dynamic posturography: aging effects and test-retest reliability. Phys Ther 91: 246-253.

24. Ford-Smith CD, Wyman JF, Elswick RK Jr, Fernandez T, Newton RA (1995) Test-retest reliability of the sensory organization test in noninstitutionalized older adults. Arch Phys Med Rehabil 76: 77-81.

25. (2015) Dr. Scholl's ${ }^{\circ}$ Custom Fit ${ }^{\circledR}$ orthotic inserts.

26. (2012) IBM Corp. Released. IBM SPSS Statistics for Windows, Version 21.0. Armonk, NY

27. Gross MT, Mercer VS, Lin FC (2012) Effects of foot orthoses on balance in older adults. J Orthop Sports Phys Ther 42: 649-657.

28. Mattacola CG, Dwyer MK, Miller AK, Uhl TL, McCrory JL, et al. (2007) Effect of orthoses on postural stability in asymptomatic subjects with rearfoot malalignment during a 6-week acclimation period. Arch Phys Med Rehabil 88: 653-660.

29. Farzadi M, Safaeepour Z, Mousavi ME, Saeedi H (2015) Effect of medial arch support foot orthosis on plantar pressure distribution in females with mild-to-moderate hallux valgus after one month of follow-up. Prosthet Orthot Int 39: 134-139.

30. Aminian G, Safaeepour Z, Farhoodi M, Pezeshk AF, Saeedi H, et al. (2013) The effect of prefabricated and proprioceptive foot orthoses on plantar pressure distribution in patients with flexible flatfoot during walking. Prosthet Orthot Int 37: 227-232.

31. Portney LG, Watkins MP (2009) Foundations of Clinical Research: Applications to Practice. Pearson/Prentice Hall, Upper Saddle River, NJ. 